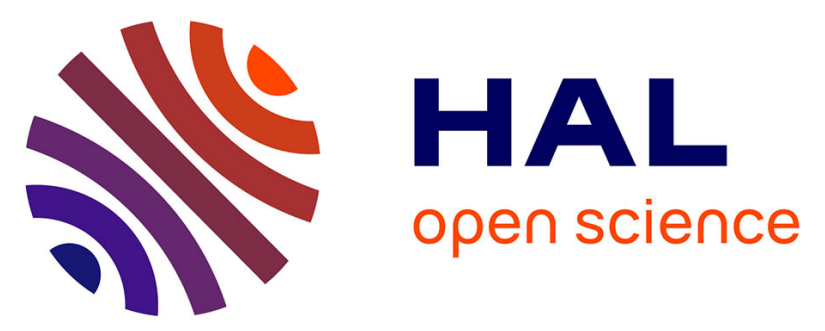

\title{
ÉTUDE PAR DIFFRACTION DES RAYONS X A TRÈS BASSES TEMPÉRATURES DES DÉPÔTS MÉTALLIQUES AMORPHES DE GALLIUM ET DE LEUR CRISTALLISATION
}

\author{
A. Bererhi, Louis Bosio, Robert Cortès, A. Defrain
}

\section{To cite this version:}

A. Bererhi, Louis Bosio, Robert Cortès, A. Defrain. ÉTUDE PAR DIFFRACTION DES RAYONS X A TRĖS BASSES TEMPÉRATURES DES DÉPÔTS MÉTALLIQUES AMORPHES DE GALLIUM ET DE LEUR CRISTALLISATION. Journal de Physique Colloques, 1975, 36 (C2), pp.C2-79-C2-82. 10.1051/jphyscol:1975216 . jpa-00216263

\section{HAL Id: jpa-00216263 https://hal.science/jpa-00216263}

Submitted on 1 Jan 1975

HAL is a multi-disciplinary open access archive for the deposit and dissemination of scientific research documents, whether they are published or not. The documents may come from teaching and research institutions in France or abroad, or from public or private research centers.
L'archive ouverte pluridisciplinaire HAL, est destinée au dépôt et à la diffusion de documents scientifiques de niveau recherche, publiés ou non, émanant des établissements d'enseignement et de recherche français ou étrangers, des laboratoires publics ou privés. 


\title{
ÉTUDE PAR DIFFRACTION DES RAYONS $X$ A TRÈS BASSES TEMPÉRATURES DES DÉPÔTS MÉTALLIQUES AMORPHES DE GALLIUM ET DE LEUR CRISTALLISATION
}

\author{
A. BERERHI, L. BOSIO, R. CORTES et A. DEFRAIN \\ Groupe de Recherche $n^{0} 4$ du C. N. R. S. \\ Physique des Liquides et Electrochimie, associé à l'Université Paris VI \\ 4, place Jussieu, 75230 Paris Cedex 05, France
}

\begin{abstract}
Résumé. - Nous montrons qu'il est possible d'obtenir des dépôts de gallium amorphe dont l'épaisseur dépasse $10 \mu \mathrm{m}$; ce qui permet d'en faire l'étude par diffraction des rayons X. Sur les courbes d'intensité réduite, le pic adjoint au pic principal apparaît très nettement accentué. La cristallisation de ces dépôts se fait vers $15 \mathrm{~K}$ en la forme métastable $\mathrm{Ga} \beta$. Des mesures de supraconduction faites simultanément permettent d'expliquer en partie, par l'existence de phases cristallines métastables, la dispersion des divers résultats publiés.
\end{abstract}

Abstract. - The possibility of obtaining amorphous gallium layers thicker than $10 \mu \mathrm{m}$ is shown ; their study by X-ray diffraction is then possible. On the reduced intensity curves, the well-marked subsidiary peak appears superimposed to the main one. The crystallisation of deposits occurs at about $15 \mathrm{~K}$ in the metastable modification $\mathrm{Ga} \beta$. Simultaneous superconducting measurements enable us to explain part of the discrepancy between the published results, by the existence of metastable crystalline gallium forms.

Introduction. - Dans des travaux antérieurs [1], nous nous étions intéressés à la cristallisation du gallium liquide qui peut être maintenu en surfusion jusqu'à $150 \mathrm{~K}$, c'est-à-dire jusqu'à $0,5 T_{\mathrm{f}}$ puisque le gallium solide, sous sa forme stable Ga $\alpha$, fond à $T_{\mathrm{f}}=303 \mathrm{~K}$. Dans ces conditions, le liquide peut cristalliser en des variétés métastables : $\mathrm{Ga} \beta$ ou $\mathrm{Ga}$ y ou $\mathrm{Ga} \delta$ ou $\mathrm{Ga} \varepsilon$.

Sur la figure 1, nous avons schématisé les diagrammes de Debye-Scherrer des variétés solides dont la structure est connue : $\mathrm{Ga} \alpha, \mathrm{Ga} \beta, \mathrm{Ga} \gamma$ et $\mathrm{Ga} \delta$ et représenté l'intensité diffusée par le liquide maintenu en surfusion à $163 \mathrm{~K}$. On remarquera l'allure inhabituelle du diagramme de diffraction du gallium liquide dont le pic principal est dédoublé.

A partir de l'état amorphe, il devrait être possible d'obtenir des états cristallins métastables analogues à ceux provenant du liquide surfondu. Nous avons entrepris d'étudier la structure et la cristallisation des dépôts amorphes relativement épais, d'une part pour ne pas être gênés par les effets dimensionnels qui jouent un rôle essentiel dans les films minces et qui ne sont pas l'objet de cette étude et, d'autre part pour nous placer dans des conditions telles que nous puissions comparer les résultats à ceux obtenus sur des échantillons massifs [2]. A la diffraction des rayons $X$, nous avons adjoint la mesure de la résistivité afin de suivre instantanément les changements de phases du dépôt et de déterminer les températures critiques de supraconduction.
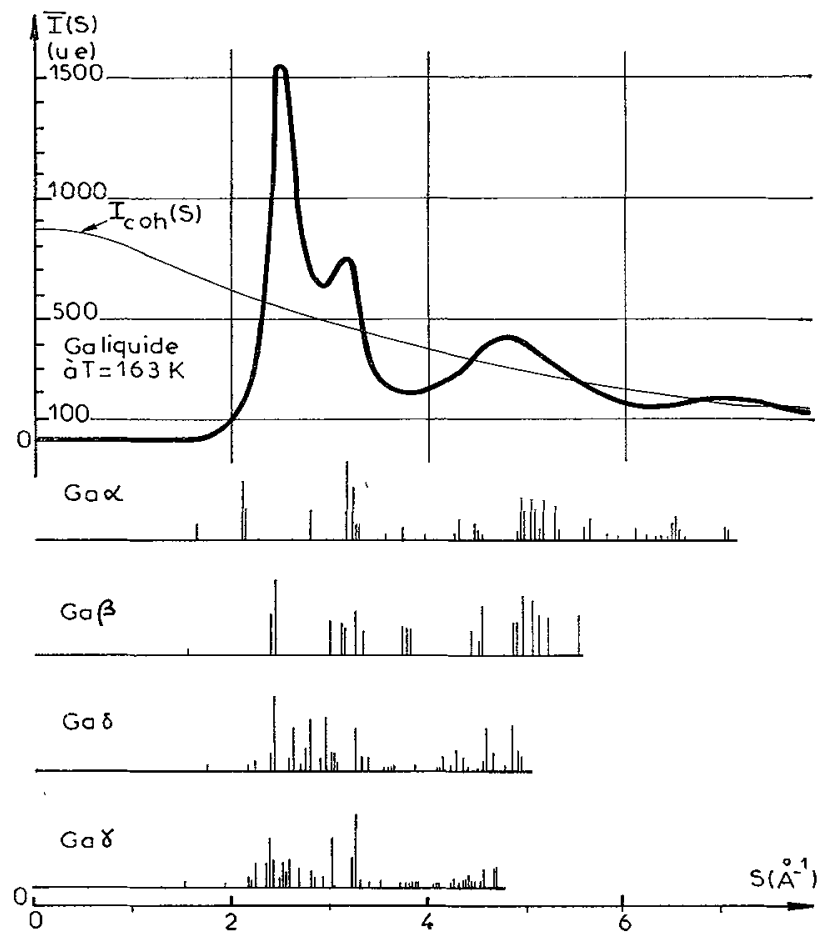

FıG. 1. - Diagrammes d'intensité diffusée par le gallium liquide et les différentes phases cristallines. 
Appareillage. - Nous donnerons une description sommaire de l'appareillage et du mode opératoire. Dans un vide de l'ordre de $10^{-8}$ torr, le métal vaporisé par bombardement électronique arrive, lorsque l'échantillon est en position basse, sur un substrat en saphir maintenu à 4,5 $\mathrm{K}$ (Fig. 2). Un passage magnétique

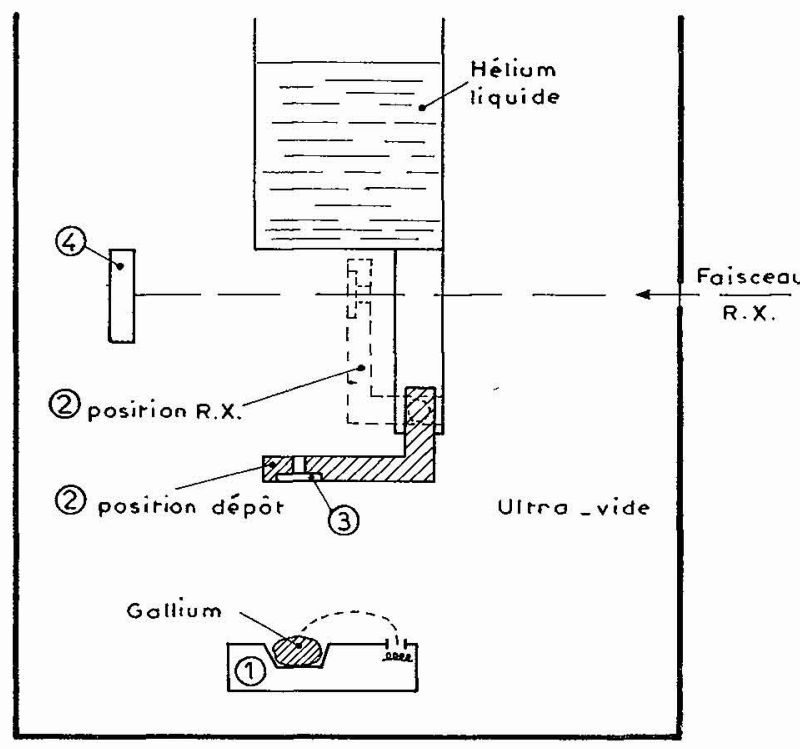

FIG. 2. - Schéma de l'appareillage. 1. Canon à électrons. 2. Porte-échantillon à orientation variable. 3. Substrat en saphir. 4. Tube compteur de rayons $X$.

tournant permet d'amener ensuite le dépôt sur le trajet d'un faisceau de rayons $X$ (rayonnement $K \alpha$ du cuivre).

A l'intérieur du bâti à vide, un compteur se déplaçant de façon continue ou au pas à pas permet de réaliser les diagrammes de diffraction et de connaître à tout instant l'état structural du dépôt [3].

Conditions d'obtention des dépôts amorphes. - Dans divers travaux, il est montré qu'il n'est pas possible de déposer à la température de l'hélium liquide des films amorphes au-delà d'une épaisseur critique, par exemple : $3000 \AA$ pour l'ytterbium [4], $50 \AA$ pour le fer [5], $600 \AA$ pour le bismuth [6], $90 \AA$ pour le béryllium [7] et $2000 \AA ̊$ pour le gallium [7, 8]. Pourtant, dans notre cas, nous pouvons obtenir des épaisseurs de gallium amorphe dépassant $10 \mu \mathrm{m}$ compatibles avec une étude de diffusion des rayons $X$ par transmission. Afin que la couche déposée reste amorphe pour les grandes épaisseurs, la condition essentielle est que pendant la formation du dépôt la température de celui-ci reste inférieure à une dizaine de Kelvin, ce que l'on vérifie dans nos expériences par les mesures supraconductrices. La vitesse d'évaporation n'a d'influence que si elle élève la température du substrat au-dessus de $8 \mathrm{~K}$; dans notre cas, la vitesse limite compatible avec cette restriction est de $1000 \AA / \mathrm{s}$. Entre $10^{-6}$ et $10^{-9}$ torr, nous n'avons pas trouvé de corrélation entre la pression qui règne à l'intérieur du bâti d'évaporation et la nature du dépôt. La plupart des couches conden- sées ont été réalisées sur de fines lamelles monocristallines de saphir. Cependant la nature du substrat n'influe pas sur la formation du métal amorphe; il a même été possible d'obtenir du gallium amorphe sur un monocristal de gallium $\alpha$; ce dernier résultat a été mis en évidence par diffraction des rayons $X$ et par des mesures de supraconduction.

Courbes d'intensité réduite du gallium amorphe. Sur la figure 3, nous présentons le diagramme d'intensité réduite des rayons $\mathrm{X}$ diffusés par le gallium

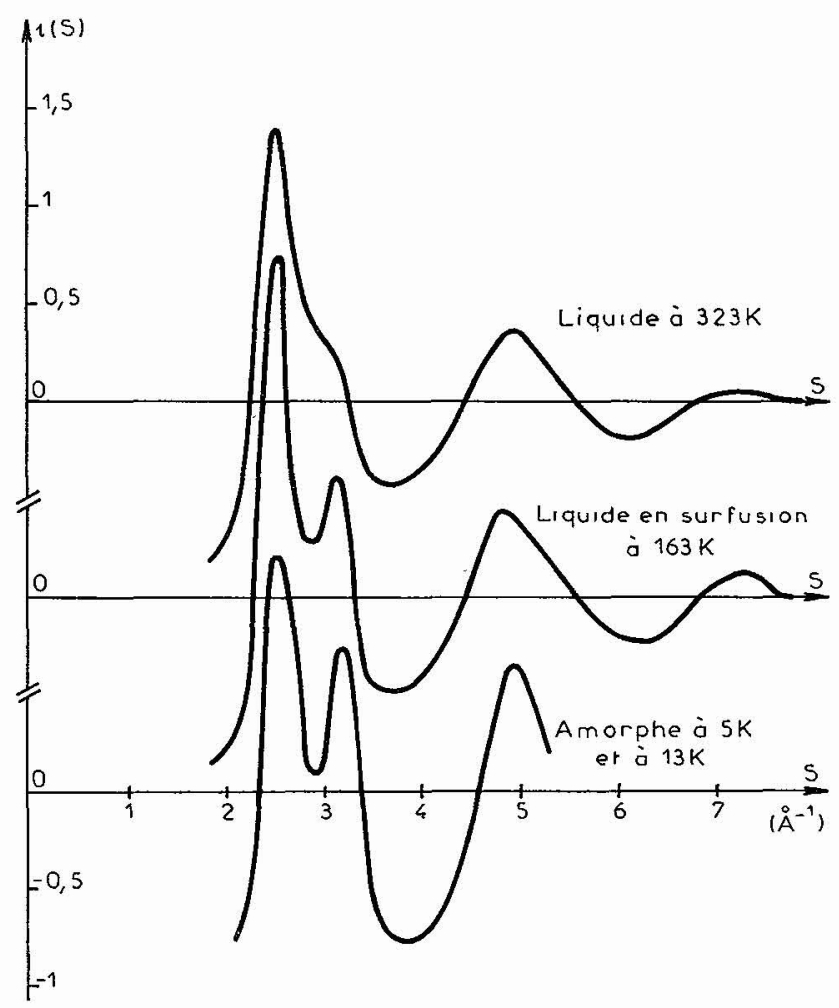

Fıg. 3. - Diagrammes d'intensité réduite diffusée par le gallium liquide et amorphe.

amorphe à $5 \mathrm{~K}$ (il est identique à celui réalisé à $13 \mathrm{~K}$ ) ; nous avons juxtaposé les diagrammes d'intensité réduite du liquide à $323 \mathrm{~K}$ et à $163 \mathrm{~K}$ en surfusion. Nous remarquons que ces courbes sont assez semblables: le pic adjoint au pic principal qui se détache de plus en plus nettement lorsque la température du liquide diminue, est encore plus accusé dans l'état amorphe. L'existence de ce pic subsidiaire, qui ne peut s'expliquer par le modèle des sphères dures, serait dû dans le cas du liquide à la forme particulière du pseudopotentiel comme l'ont montré Badiali et Regnaut [9]. On peut penser que cette explication reste valable pour le gallium amorphe.

Transformation des dépôts amorphes. - Ces dépôts de gallium amorphe ont une température critique de supraconduction de $8,2 \pm 0,2 \mathrm{~K}$ (Fig. 4) ; au réchauffement, on observe entre 15 et $20 \mathrm{~K}$, une décroissance de 


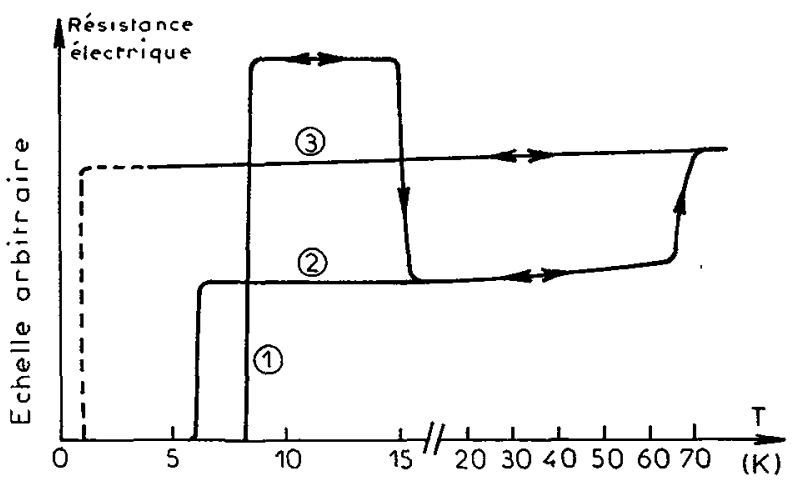

FIG. 4. - Variation de la résistance électrique des dépôts en fonction de la température. 1. Gallium amorphe. 2. Gallium $\beta$. 3. Gallium $\alpha$.

la résistance. En refroidissant de nouveau l'échantillon, la transition supraconductrice se produit à $6,0 \mathrm{~K}$ : un diagramme de rayons $\mathrm{X}$ montre que le dépôt est maintenant cristallisé et l'indexation des raies de diffraction indique que c'est la variété cristalline $\mathrm{Ga} \beta$ monoclinique qui est apparue. La température de cristallisation du gallium amorphe dépend des dimensions de l'échantillon : $15 \mathrm{~K}$ est la température minimale à laquelle se fait la cristallisation pour les échantillons les plus épais indépendamment de la nature du substrat (saphir, gallium cristallisé). Ces résultats montrent qu'en dessous de $15 \mathrm{~K}$, la mobilité des atomes est pratiquement nulle. Un réchauffement ultérieur de $\mathrm{Ga} \beta$ conduit à sa transformation vers $60 \mathrm{~K}$, en la forme stable, orthorhombique, Ga $\alpha$ (Fig. 5). Comme la température de supraconduction de $\mathrm{Ga} \alpha$ est $1,08 \mathrm{~K}$, on n'observe plus de transition supraconductrice au-dessus de $4,5 \mathrm{~K}$.

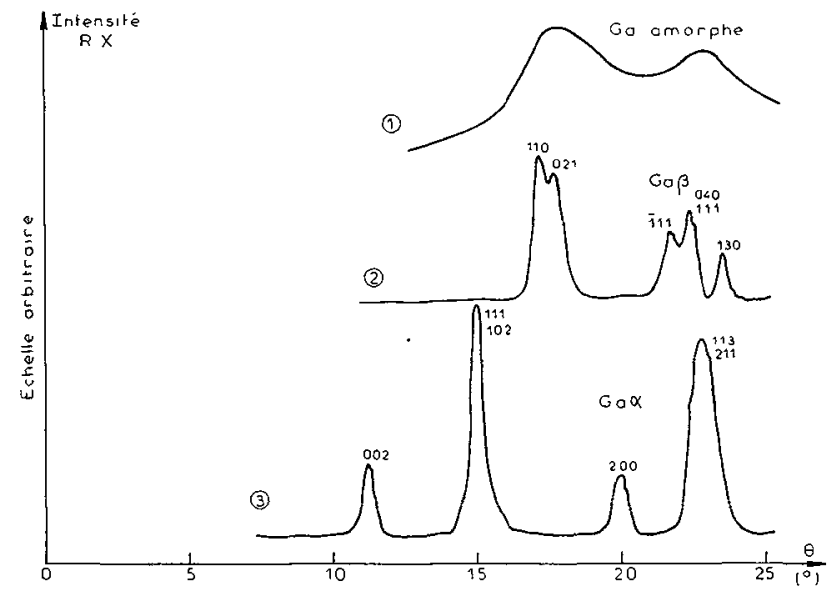

FIG. 5. - Diagrammes de diffraction des rayons $\mathrm{X}$ montrant l'évolution des dépôts amorphes. 1. Immédiatement après la condensation. 2. Immédiatement après un réchauffement audessus de $15 \mathrm{~K}$. 3. Immédiatement après un réchauffement au-dessus de $60 \mathrm{~K}$.

Supraconduction du gallium en couches minces. Nos mesures apportent des précisions pour interpréter les divers résultats concernant la supraconduction du gallium en couches minces. De nombreux travaux ont été consacrés aux transitions supraconductrices des films de gallium déposés à très basse température et l'on peut voir sur le tableau I, la grande dispersion des

\section{Tableau I}

Transitions supraconductrices des films de gallium

\begin{tabular}{|c|c|c|c|}
\hline$T_{\mathrm{c}}$ & $2 \Delta_{0} / K_{\mathrm{b}} T_{\mathrm{c}}$ & \multicolumn{2}{|c|}{$\begin{array}{l}\text { Procédé } \\
\text { et Références }\end{array}$} \\
\hline 6,21 & $3,95-4,02$ & E. T. & {$[10]$} \\
\hline $6,26-6,31$ & & I. R. & [11] \\
\hline 6,3 & & Rés. & [12] \\
\hline 6,4 & 3,8 & E. T. & [13] \\
\hline $6,55-7,09$ & 3,94 & I. R. & [11] \\
\hline reproductible & 3,9 & E. T. & [14] \\
\hline $7,74-7,87$ & 3,87 & I. R. & [11] \\
\hline 7,9 & 4,1 & E. T. & [13] \\
\hline $8,05-8,45$ & 4,35 & E. T. & [11] \\
\hline 8,3 & 4,65 & E. T. & [15] \\
\hline 8,4 & & Rés. & [12] \\
\hline 8,4 & 4,2 à 4,5 & E. T. & {$[13,14,16]$} \\
\hline 8,47 & $4,50-4,52$ & E. T. & [10] \\
\hline 8,56 & 4,51 & E. T. & [17] \\
\hline
\end{tabular}

E. T. Effet tunnel.

I. R. Absorption infra-rouge.

Rés. Résistivité.

résultats due, d'une part, aux incertitudes sur $T_{\mathrm{c}}$ liées aux variations de ce terme avec l'épaisseur du dépôt [18] et d'autre part, à l'existence de variétés cristallines métastables dont les températures critiques sont 6,0 6,9-7,6 et 7,85 K comme nous l'avons montré par ailleurs [19]. Cette dernière cause de la dispersion des résultats a été ignorée par certains auteurs et a entraîné des confusions dans l'interprétation des transitions supraconductrices du gallium puisque, à notre connáissance, aucun contrôle de la structure du dépôt n'était réalisé conjointement avec les mesures de résistivité. Ce travail montre que les températures critiques voisines de $8,2 \mathrm{~K}$ sont relatives au métal amorphe et celles voisines de $6 \mathrm{~K}$ concernent la variété $\mathrm{Ga} \beta$. Il ne nous a pas été possible jusque-là de mettre en évidence les autres phases métastables par diffraction des rayons $X$, bien que les courbes de résistivité aient montré dans quelques cas des transitions supraconductrices à des températures différentes de celles situées à $6,0 \mathrm{~K}$ et à $8,2 \mathrm{~K}$.

Pour conclure, nous retiendrons qu'il est possible d'obtenir du gallium amorphe en couche épaisse, d'en déterminer l'arrangement atomique à courte distance par diffraction des rayons $X$ et d'étudier la cristallisation en formes métastables. L'existence de ces phases métastables permet d'expliquer en partie la dispersion des résultats de la littérature concernant les mesures de supraconduction des couches de gallium. 


\section{Bibliographie}

[1] Bosio, L., Defrain, A. et Epelboin, I., J. Physique 27 (1966) 61 ;

Bizid, A., Bosio, L., Curien, H., Defrain, A. et Dupont, M., Phys. Stat. Sol. A 23 (1974) 135.

[2] Bosio, L., Defrain, A. et Dupont, M., J. Chim. Phys. 68 . (1971) 542.

[3] Bererhi, A., Bosio, L., Cortes, R. et Defrain, A., Electrodep. Surf. Treat. 2 (1974) 455.

[4] Lazarev, B. G., Kuz'menko, V. M., Sov. Phys, Dokl. 14 (1969) 77.

[5] Lazarev, B. G., Kuz'menko, V. M., Sov. Phys. J. E. T. P. Lett. 10 (1969) 165.

[6] Lazarev, B. G., Kuz'Menko, V. M., Sudovtsoy, A. I. et Mel'nokov, V. M., Sov. Phys. Dokl. 15 (1971) 846.

Komnik, Yu. F., Belevisev, B. I. et Yatsuk, L. A., Sov. Phys. J.E. T. P. 36 (1973) 1177.

[7] Lazarev, B. G., Kuz'menko, V. M., Sudovtsov, A. I. et Mel'NiKov, V. M., Fiz. Met. Metal. 32 (1971) 52.

[8] Belevtsev, B. I. et Komnik, Yu. F., Sov. Phys. Solid State 14 (1973) 2759.
[9] Badiali, J. P. et Regnaut, C., Phys. Stat. Sol. 63B (1974) 555.

[10] Minnigerode, G. V. et Rothenberg, J., Z. Phys. 213 (1968) 397.

[11] Harris, R. E. et Ginsberg, D. M., Phys. Rev. 188(1969) 737.

[12] Buckel, W. et Hilsch, R., Z. Phys. 138 (1954) 109.

[13] Combe, R. W., Abeles, B. et Weisbarth, G. S., Phys. Rev. Lett. 18 (1967) 336.

[14] Wuhl, H., Jackson, J. E. et Briscoe, C. V., Phys. Rev. Lett. 20 (1968) 1496.

[15] Vashishta, P. et Carbotte, J. P., J. Low Temp. Phys. 10 (1973) 551.

[16] Chen, T. T., Chen, J. T., Leslie, J. D. et Smith, H. J. T., Phys. Rev. Lett. 22 (1969) 526.

[17] Zavaritskit, N. V., Sov. Phys. J.E. T. P. 30 (1970) 412 et Usp. Fiz. Nauk. 108 (1972) 241.

[18] Granevist, C. G. et Cleason, T., Phys. Cond. Matter 18 (1974) 79.

[19] CorTes, R., Thèse d'état, Paris 1974 (CNRS no AO 9543). 